\title{
BMJ Open Using qualitative insights to change practice: exploring the culture of antibiotic prescribing and consumption for urinary tract infections
}

\author{
Sinead Duane, ${ }^{1}$ Christine Domegan, ${ }^{2}$ Aoife Callan, ${ }^{1,3}$ Sandra Galvin, ${ }^{1}$ \\ Martin Cormican, ${ }^{4,5}$ Kathleen Bennett, ${ }^{6}$ Andrew W Murphy, ${ }^{1}$ Akke Vellinga ${ }^{1,4}$
}

To cite: Duane $\mathrm{S}$,

Domegan C, Callan A, et al. Using qualitative insights to change practice: exploring the culture of antibiotic prescribing and consumption for urinary tract infections. BMJ Open 2016;6:e008894. doi:10.1136/bmjopen-2015008894

- Prepublication history for this paper is available online. To view these files please visit the journal online (http://dx.doi.org/10.1136/ bmjopen-2015-008894)

Received 25 May 2015 Revised 9 November 2015 Accepted 10 November 2015

CrossMark

For numbered affiliations see end of article.

Correspondence to

Dr Sinead Duane;

sinead.duane@nuigalway.ie

\section{ABSTRACT}

Objectives: The aim of this paper is to explore the culture of antibiotic prescribing and consumption in the community for urinary tract infections (UTI) from the perspective of the general practitioners (GPS) and community member.

Design: Indepth interviews were conducted with GPs, and focus groups were held with community members.

Setting: General practice and community setting. Participants: 15 GPs practising in rural and urban locations in Ireland participated in the indepth interviews. 6 focus groups $(n=42)$ with participants who had direct or indirect experiences with UTI were also undertaken.

Results: The decision to prescribe or consume an antibiotic for a UTI is a set of complex processes including need recognition, information search and evaluation processes governed by the relationship and interactions between the GP and the patient. Different GP and patient decision-making profiles emerged emphasising the diversity and variety of general practice in real-life settings. The GP findings showed a requirement for more microbiological information on antibiotic resistance patterns to inform prescribing decisions. Focus group participants wanted a conversation with the GP about their illness and the treatment options available.

Conclusions: Collectively, this research identified the consultation as a priority intervention environment for stimulating change in relation to antibiotics. This paper demonstrates how qualitative research can identify the interacting processes which are instrumental to the decision to prescribe or consume an antibiotic for a suspected UTI. Qualitative research empowers researchers to investigate the what, how and why of interventions in real-life setting. Qualitative research can play a critical and instrumental role in designing behavioural change strategies with high impact on practice. The results of this research were used to design a complex intervention informed by social marketing.

Trial registration number: NCT01913860; Preresults.

\section{Strengths and limitations of this study}

- The combination of qualitative research, theoretical and decision-making social marketing frameworks ensured that the research findings could be used to design an intervention which met the needs of both the general practitioner (GP) and the patient.

- This research not only provided the foundations to design complex interventions, it also contributed to the refining of the primary and secondary outcomes within the randomised control trial and the recruitment strategy.

- As with all qualitative research, the sample size is small, however, the level of detail emerging is of great value, and the methodology described can be replicated in other settings.

- Small incentives (gift vouchers) were used to compensate the participants of this research which could have led to selection bias.

- A patient's choice of GP is limited by geography, and this may impact on their attitudes and behaviours towards consuming antibiotics for a urinary tract infection.

\section{BACKGROUND}

Antibiotic resistance (ABR) is a global public health issue, and the overprescription and consumption of antibiotics in the community is a main driver. ${ }^{1-3}$ Interventions to address this issue are often aimed at the general public and fail to acknowledge the decisions made by specific groups such as general practitioners (GPs) or their patients. ${ }^{4-6}$ These decisions become important as the prescription of antibiotics goes beyond a simple or uniform decision, to prescribe or not. ${ }^{7}$ This simple decision mindset downplays the power relations inherent in a GP-patient consultation and the wider social structures that shape antibiotic prescription and consumption patterns. For example, GPs within the Irish healthcare 
system see a mixture of private fee-paying patients and patients covered under the General Medical Services (GMS) scheme. Fee-paying patients pay between $€ 40$ and $€ 60$ to consult their GP while GMS patients receive free healthcare with a co-payment of approximately $€ 1.50$ per prescription. Approximately $30 \%$ of the Irish population is entitled to the GMS scheme. ${ }^{8}$ Payment of consultations may be one factor that influences the expected outcome of the consultation, however, others also exist.

Qualitative research has contributed to our understanding of the culture of prescribing antibiotics for infections such as lower respiratory tract infections ${ }^{9-11}$ and the hospital setting; ${ }^{12}$ leading to the development of successful behavioural interventions. More research needs to be undertaken to understand the decisionmaking processes contributing to the continued prescription and consumption of antibiotics for urinary tract infections (UTIs). To identify and comprehend strategies for change, antibiotic prescribing and consumption for UTI is best analysed within the reality in which the behaviours exist. ${ }^{13}$ Interrogating and integrating these behaviours and decision-making processes can contribute to the design of successful long-term behavioural change strategies.

The aim of this paper is to explore the culture of antibiotic prescribing and consumption in the community for UTI from the perspective of the GP and community member. This paper demonstrates how qualitative research can identify the interacting processes which are instrumental to the decision to prescribe or consume an antibiotic. These findings can be used to design complex interventions to facilitate change. UTI is the second most common infection in primary care. ${ }^{14-18} \mathrm{~A}$ recent study of antibiotic prescribing in primary care for UTI in Ireland identified that only 55\% of antibiotic prescriptions could be interpreted as appropriately targeted when evaluated against the laboratory report on the urine sample. ${ }^{1}$

\section{METHOD}

\section{Participants}

Fifteen indepth interviews with GPs and 6 community focus groups with 42 participants were conducted in 2013. Purposeful non-probability sampling was used to recruit all indepth interview and focus group participants. To recruit GPs, 30 postal invitations were sent to members of an established GP network consisting of over 170 GPs. All GPs selected practiced outside the proposed intervention catchment area, but had similar characteristics to it including a mixture of GPs practising in urban and in rural locations. In two cases, two GPs of different gender were recruited from the same practice. All GPs were assumed to be able to improve their antibiotic prescribing practices, ${ }^{1}$ no further selection criteria was applied. Invitations were followed-up with a telephone call to confirm an interview. Table 1 summaries the characteristics of the participating GPs. Participants

\begin{tabular}{ll}
$\begin{array}{l}\text { Table } 1 \text { General practitioners (GP) indepth interview } \\
\text { characteristics }\end{array}$ & Count $(n=15)$ \\
\hline Age (years) & 6 \\
$30-39$ & 4 \\
$40-49$ & 3 \\
$50-59$ & 2 \\
$60+$ & Count $(n=14)$ \\
Number of years practising as a GP & 4 \\
$\leq 5$ & 4 \\
$6-15$ & 6 \\
$\geq 16$ & Count $(n=15)$ \\
Number of GPs in your practice & 3 \\
Single handed & 8 \\
$2-3$ & 3 \\
4 & 1 \\
$>4$ & Count $(n=15)$ \\
Area in which your practice is based & 3 \\
City & 5 \\
Town & 1 \\
Village & 6 \\
Countryside &
\end{tabular}

ranged in age and practice size. The number of years' experience as a GP ranged from 3 to 37 .

Two focus groups were recruited from a rural location and four from an urban. Forty-two participants were recruited and included those who had direct and indirect experience of a UTI. A gatekeeper recruitment strategy was used to contact participants. Gatekeepers were identified as people who had access to the study population and had a prior relationship with them, for example, community group leaders. Gatekeepers were contacted via email and/or telephone; they, in turn, nominated members of their network to participate who fulfilled the eligibility criteria. Participants were eligible if they were over 18 years of age and able to give informed consent. Participants ranged in age from 18 to over 70 years, however, participants of similar ages were recruited to each group to identify if there were any cultural differences between the age groups. Exclusion criteria included anyone who had recently suffered from a complicated UTI, had insufficient command of the English language (spoken and written), and were pregnant or breastfeeding. Breastfeeding mothers often suffer from UTI due to hormonal changes. This would result in a different discussion than was intended for the study, therefore, they were excluded.

The gatekeepers initiated contact with their network and assisted in arranging the focus groups to ensure it was convenient to participate. Five focus groups were conducted with females, as almost half of all females experience at least one UTI episode during their lifetime. ${ }^{14}$ One focus group was conducted with males only as they are less likely to experience UTI, therefore, their experiences of consulting with a GP, and attitudes towards consuming antibiotics, may be different. Male and female groups were also separated to avoid potential 
Table 2 Focus group participant characteristics

\begin{tabular}{ll} 
Age (years) & Response count $(\mathrm{n}=42)$ \\
$<30$ & 11 \\
$30-39$ & 11 \\
$40-49$ & 2 \\
$\geq 50$ & 18 \\
Medical card status & Response count $(\mathrm{n}=42)$ \\
GMS scheme patient & 15 \\
Area in which they lived & Response count $(\mathrm{n}=42)$ \\
City & 18 \\
Countryside & 12 \\
Town & 6 \\
Village & 6 \\
Have you ever had a UTI? & Response count $(\mathrm{N}=38)$ \\
Yes, one & 5 \\
Yes, several & 7 \\
None & $26^{*}$ \\
\hline
\end{tabular}

${ }^{*}$ Ten of the participants were male and therefore less likely to have experienced a UTI.

GMS, General Medical Services; UTI, urinary tract infection.

embarrassment due to the topic being discussed. Gender differences may impact on intervention design and, therefore, needed to be investigated. Over one-third of participants were GMS patients (received free healthcare) in line with the national average. Each focus group comprised of 5-10 participants. Table 2 summarises the characteristics of the focus group participants.

The overall recruitment strategy is summarised in table 3.

Recruitment continued until theoretical saturation was achieved. All participants were remunerated (gift voucher) to participate in this research.

\section{Procedures}

The first author (SD) conducted all indepth interviews and facilitated all focus groups. The third author (AC) acted as the second facilitator in all focus groups. All indepth interviews and focus groups were led using topic guides. These questions which were used to guide the discussion, however, were flexible and adaptable to ensure the conversation was not constrained. A summary of key questions is provided in table 4. Discussion was limited to the community healthcare setting, and focused on knowledge, attitudes and awareness of the role of antibiotics in general and, specifically, the decision-making processes for treatment of UTIs. The topic guides were informed by a literature review and in consultation with a multidisciplinary team of experts. Two decision-making theories guided the development of the topic guide and the analysis process; Trans Theoretical Model (TTM) ${ }^{19}$ and the Buyer Behaviour and Decision Making Model. ${ }^{20}$ These theories were adopted to understand the inter-relating contextual factors and processes which contributed to prescription and consumption decision-making. The TTM focused on the decision-making process of the GP to prescribe and their readiness to change. The model has five stages (Precontemplation, Contemplation, Preparation, Action and Maintenance), and is the most used theory by health researchers to identify and tailor interventions to facilitate behavioural change. ${ }^{21}$ The Buyer Behaviour and Decision Making Model also has five stages (Need Recognition, Information Search, Evaluation of Alternatives, Purchase Decision and Purchase Evaluation). This model evaluated how, when and why someone consults with a GP, and how they evaluate the outcome. Both models captured direct and indirect factors which influenced decision-making.

Three pretest indepth interviews and two pretest focus groups were undertaken prior to the start of data collection. Minor changes were made as a consequence such as plain English proofing and reordering of questions.

Indepth interviews were conducted in the GP's practice and lasted between $30 \mathrm{~min}$ and $1 \mathrm{~h}$. Fourteen were audio recorded, one participant declined to be audio recorded, so in this case, only hand written notes were available. Focus groups were conducted in locations convenient to participants and lasted approximately $1 \frac{1 / 2}{\mathrm{~h}}$.

Prior to participation in this research, all participants were asked to complete a brief profile survey, and written consent was obtained.

\section{Data analysis}

After each focus group, a debriefing session was held to discuss the session and emerging themes. Digital recordings were transcribed verbatim; transcripts were re-read and coded aided by manual coding and Nvivo V.10. In applying an analytical perspective to the qualitative data analysis, we adopted a realist perspective, which emphasises the importance of context for interpreting reality, and that the phenomena under investigation are complex. $^{22}$

Table 3 Summary of sampling and recruitment strategy

\begin{tabular}{lll}
\hline Sample & Recruitment strategy & Sample size \\
\hline Females aged 18 years and over & Via local gatekeeper groups=senior citizen social clubs & 2 pretest groups \\
& and young mothers groups & 5 focus groups \\
Males aged 18 years and over & Via local gatekeeper groups=Men's Sheds users & 1 focus group \\
GPs & Members of an established GP research network. & 3 pretest interviews \\
& Recruited via invitation letter and follow-up by phone call & $\mathrm{N}=15$ \\
& to the practice &
\end{tabular}


Table 4 Summary of key questions discussed within this research

\section{GP interview questions}

Section 1: Usual practice for treating a UTI

Can you talk me through how you would normally diagnose someone with a UTI? What treatment do you recommend, how do you make this choice? Please describe the role of the patient in the diagnosis?

Section 2: Antibiotics

Overall, what are your views on prescribing antibiotics?

Positive/negative aspects? Do these views change for a patient with UTI? Have you ever received any guidelines on prescribing antibiotics? Can you remember what the guidelines are? Do they include UTI? How did you feel about using this guidelines in practice?

Section 3: Antibiotic resistance

Are there any adverse side effects to prescribing antibiotics? Do you know what the antimicrobial resistance patterns are in your area?

\section{Section 4: Intervention design}

Discussion of possible strategies to facilitate changing their attitudes and behaviours towards prescribing antibiotics for UTI

\section{Focus group questions}

Section 1: General health and GP consultations

Activity to establish participants' health-seeking behaviours and current relationship with GPs

\section{Section 2: Awareness of antibiotics}

Can you explain to me what an antibiotic is? Have you been prescribed any kind of antibiotic in the past year? Did you ask your GP/doctor any questions relating to the prescription? Can you describe the benefits and consequences of taking an antibiotic?

Section 3: Urinary tract infections experiences and associations Scenario-based exercise describing symptoms experienced by a patient with typical UTI. Discussions of personal experiences of having a UTI and the actions taken throughout the illness.Has anyone here ever experienced a UTI, or known someone who has had one-what words or phrases would you associate with it? Please describe the steps that you go through when deciding to go to see your GP doctor key priorities.Scenario-based exercise to discuss association between UTI illness and antibiotic

Section 4: Antibiotic resistance

Have you ever heard of the term antibiotic resistance? What does it mean to you? In what context did you hear it?

Section 5: Intervention design

Discussion of possible strategies to facilitate changing their attitudes and behaviours towards consuming antibiotics for

UTI

GP, General Practitioners; UTI, urinary tract infections.
Thematic data analysis was concurrent with data collection and followed Braun and Clarks ${ }^{23}$ six-step process. We integrated, coded and thematically analysed both data sets using an interpretive approach and a coding scheme derived both from the research aim and from issues that emerged during data generation and early analysis. The TTM and Buyer Behaviour and Decision Making Model were used to inform the initial codes. Throughout the process of analysis, the data was constantly compared to identify the underlying themes within the data.

\section{RESULTS}

The results of this research focus on knowledge of ABR and the factors which affect the decision to consult a GP, the diagnosis of a UTI, and how it is treated.

\section{Knowledge of antibiotic resistance}

GPs are knowledgeable of the definition of ABR and the consequences of it, however, their discussion of ABR focused on the longer term societal impact. GPs accepted that antibiotics were overprescribed contributing to the spread of ABR, but are also a necessary part of a modern healthcare system. They believed that other sectors, such as vets and agriculture, were also part of the problem.

\section{GPs role in creating awareness}

All but one GP agreed it was the GPs responsibility to discuss the issue of ABR with their patients. However, many did not engage in this conversation within every consultation. All GPs felt they needed evidence from the microbiology laboratory to support this discussion. In addition, GPs perceived discussing ABR with patients as time consuming which was a major concern.

Probably the way the practice works here it's so busy that they're not given an opportunity to kind of discuss it, you know. (GP 12)

The patient also needed to be willing to listen, this was not the case in all instances.

The GPs who believed they were more prudent prescribers, had already integrated a conversation about ABR into the consultation. They used it as a justification for not prescribing an antibiotic, or using a delayed prescribing strategy. Their comfort with this conversation 
developed over time, and by focusing on the short-term benefits the message seemed to be accepted.

Certainly sometimes I use it as leverage to kind of try to avoid giving prescriptions. (GP 13)

No GP discussed ABR specifically with the patient once they had prescribed an antibiotic.

Because I would usually have the decision made myself that this person needs an antibiotic or they don't... (GP 12)

\section{Patient knowledge of ABR}

By contrast, when asked directly focus group participants found it difficult to define $A B R$, instead making reference to becoming 'immune' to antibiotics if you do not consume them as instructed. Overall, focus group participants lacked an awareness about the lasting consequence of ABR. They were unaware that antibiotics would 'run-out' in the future if not protected. Focus group participants believed this type of information would encourage them to question their consumption. However, messages needed to be simple and relevant to them.

\section{Decision to consult a GP}

Focus group participants were aware of the symptoms of a UTI and the discomfort associated with it. Prior to consulting a GP with UTI symptoms, half the focus group participants described trying home remedies such as 'flushing' the UTI out with water and cranberry juice.

Start by trying to deal with it on your own and then if it really doesn't go away, go to the doctor. (FG 1)

Advice was often sought from close family members (usually mothers) and pharmacists relating to how to manage the UTI. If symptoms persisted and were deemed severe, participants would then decide to consult a GP.

Other participants used past experience to assess their need to consult a GP. Some who had experienced UTI in the past, and associated their present symptoms with a UTI consulted the GP immediately expecting antibiotic treatment and a shorter duration of illness.

All the time you hear people saying it. They're going to the doctor to ask them to get antibiotics. They expect it from the consultation. (FG 1)

Only one participant described a situation whereby she refused an antibiotic for a UTI until further tests were undertaken.

Like I had a UTI during the year and I didn't want an antibiotic and she sent away the sample and rang me at work and said you have to take an antibiotic and I said why? Then she said there's blood in your urine. You have to take one and come back in if I needed to discuss it with her. (FG 2)
This participant's mother had been reluctant to give her antibiotics as a child, therefore, she had a cautious approach to consuming them. The participants indicated they did not want to consume antibiotics if they could avoid it.

Trust and value for money were also important factors when deciding to consult with a GP. GPs were deemed knowledgeable, and participants trusted their diagnosis if symptoms persisted, and wanted reassurance that their symptoms would not worsen.

In addition to trust, value for money was identified as a crucial factor when deciding to consult for a minor illness, particularly for fee-paying patients. Value for money was evaluated against severity of symptoms and/or consulting with the GP over a number of ailments at once. It may be linked to receiving an antibiotic, however, reassurance that symptoms will not worsen was also highly valued depending on the patient profile.

Whether or not to spend 50 quid to bring the child to the doctor and yourself to the doctor or to put food on the table. It's a hard choice... (FG 6)

Value, ultimately linked to satisfaction was also associated with consultation duration, closeness of relationship and communication between the GP and the patient and not necessarily leaving the practice with a prescription for an antibiotic.

He listens to you. Some doctors don't listen to you. They just brush you by. He listens to you and takes you seriously and do something about it. (FG 5)

More health-conscious individuals, such as the young mothers, sought reassurance that they were not suffering from a more serious condition, and that they could return to their daily lives as soon as possible rather than an antibiotic.

As long as I found some way of alleviating whatever pain I was in I don't care whether I got a prescription or not. (FG 3)

GPs stated they did not treat private and GMS patients differently, however, they were conscious of patients receiving value for money from the consultation.

Yeah, I mean I suppose the GMS patients would present more frequently and earlier than the private patients purely for financial...You know, the private patient would certainly have done the cystopurin and the cranberry juice first and would present later, yeah. (GP 12)

\section{Diagnosing and treatment of a UTI}

The treatment of UTI centred around two decisionmaking processes, first, whether to prescribe an antibiotic or not, and second the type of antibiotic prescribed. 
Diagnosis of a UTI

UTI consultations are common and a relatively simple consultation which GPs did not want to complicate or elongate.

Great. In and out in two minutes...I think it's something that's very straightforward... (GP 6)

GPs believed UTIs were easy to treat, and all GPs interviewed asked patients to describe their symptoms, some followed this conversation with a dip stick test. If the dipstick is negative but the symptoms are suggestive of a UTI, a GP may choose to prescribe anyway.

I think if the symptoms are very suggestive, it doesn't necessarily mean that they don't have an infection. So, I suppose if they had normal urinalysis I might look at weighing up the pros and cons of what treatment to prescribe. (GP In 3)

In this instance of uncertainty, a GP will choose whether to prescribe an antibiotic or not. Some of the GPs prescribed an antibiotic if they believed the symptoms suggested a UTI. In other cases, if the patient had already delayed consulting with the GP, the symptoms were perceived as severe and non-responsive to other remedies they was more inclined to prescribe.

A sense of guilt arose when private patients presented, as they were paying a fee for the consultation, and even GPs who were comfortable not prescribing or delaying prescriptions perceived the private patient to expect a prescription.

I think it's much more difficult not to prescribe with a private patient because they're coming in and paying $€ 45$ for a consultation and they don't expect just something that can get over the counter. I think they're probably as willing to defer the prescription as a public patient but I think I probably would be more likely to give a delayed prescription to them than no prescription at all... (GP 13)

Consultations within general practice are often unpredictable, with time constraints impacting on what can be discussed. The perceived ease of diagnosing a UTI coupled with external pressures related to duration of consultation often resulted in a quick diagnosis. Patients wanted to get back to health quickly.

\section{Antibiotic treatment of UTI}

Both GPs and focus group participants agreed that, in general, GPs were prescribing less antibiotics. However, GPs have not changed their behaviour for UTI. They believe antibiotics are a necessary treatment for patients experiencing a UTI. Antibiotic treatment for UTI is usually empirical due to a delay between the consultation and microbiological analysis results confirming a UTI.

So, rather than sitting in the patient, I would treat them with a broad spectrum antibiotic and I would send a sample off for a culture and sensitivity and we'd see how we were fixed when that would come back whether we were on the right antibiotic or not and that's basically... (GP 1)

GPs preferred to treat empirically due to the discomfort experienced by the patient, and to reduce reconsultations. Once they have decided to prescribe an antibiotic it is difficult to change their minds. However, if a GP was unsure of the diagnosis, multiple factors influence the decision to treat a UTI with an antibiotic, beyond the illness itself. Additional considerations include severity of symptoms, personal circumstances, previous experiences (GP and patient), the GP's general attitude to treating UTI, and grey areas whereby symptoms are presented but there is no evidence of a UTI. Any combination of factors could influence the outcome of the consultation.

For GPs who did not wish to prescribe, negative dip stick (urine test) results, coupled with the lag time with receiving laboratory results, present GPs with an opportunity to delay antibiotic treatment until results are known. This conversation was easier with patients who preferred not to take antibiotics. However, there were cohorts who wanted to get well quickly and wanted an antibiotic to treat their symptoms.

Even a GP who has a well-established reputation for not prescribing antibiotics, accepts that prudent prescribing is a long-term strategy whereby patients may only see the benefits in the future.

No. Now, twenty years on people begin to think maybe we're right (GP 8)

It is also acknowledged that not all patients share the same views on antibiotic consumption and, therefore, patients also need to be willing to change their perspectives.

Well, first of all they've been used to the pattern of getting them down through the years...They feel they need to get something and to get over the infection as quick as possible. So, that's part of the problem. (GP 9)

\section{Antibiotic prescribing preferences}

The GPs had spent little time reflecting on the influences on their antibiotic prescribing preferences with many GPs prescribing the same antibiotic for UTI routinely. However, some patients requested an antibiotic and, in some cases, even indicated the treatment they thought was required.

GPs rarely received formal feedback on their prescribing, few were knowledgeable on local resistance patterns, and their antibiotic prescribing preferences were, instead, formed through habit (prescribing the same antibiotic routinely), anecdotal evidence from observing patients, and the laboratory results of individual patients. In a few cases, GPs cited observing patterns within their patients which suggested that there was increased resistance to trimethoprim (a type of antibiotic) in the 
community. This knowledge encouraged the GPs to switch to alternatives. The GPs were aware of guidelines but rarely cited as the primary reason for choosing a particular antibiotic treatment.

\section{DISCUSSION}

The diversity and complexity of factors contributing to the culture of antibiotic prescribing and consumption for UTI in the community is evident within the findings from our research. Few qualitative studies have discussed the culture of antibiotic prescribing and consumption from the perspective of the GP and the patient. This research highlights how difficult it is to capture the complex interactions which contribute to antibiotic prescribing for UTI. These interactions take place within the consultation, and like other studies, our findings highlight the important role of the consultation encounter when deciding to prescribe, particularly if prudent prescribing is a desired outcome. ${ }^{24}$

Overall, GPs are aware of the consequences of ABR and have taken steps to improve their prescribing behaviours particularly when treating colds and flu. Unlike other areas in general practice where improvements have been made to antibiotic prescribing practices, ${ }^{11}$ the GPs within this research viewed UTI differently, instead believing that antibiotics are a necessary treatment for UTI. This mindset and the perceived ease of the UTI consultation, have contributed to GPs not questioning their prescribing decisions. Support from microbiological laboratory may interrupt this culture and encourage GPs to question their prescribing decisions. ${ }^{5}$

The findings outline at least two distinct GP decisionmaking perspectives, the 'habitual' and 'questioning' prescriber both representing different views on the culture of prescribing. The 'habitual prescriber' treats all UTIs with an antibiotic if they believe symptoms are consistent with a UTI, particularly if the patient has tried to manage the symptoms themselves before consulting. This GP is in the precontemplation stage of changing their prescribing behaviour. They need to be convinced of the necessity of change through scientific evidence. The 'questioning prescriber', recognises there is a grey area when diagnosing a UTI, these GPs are more willing to question the need for an antibiotic depending on the symptoms presented to them by the patient and the dipstick results. These GPs are in the contemplation or action stages of change. Evidence linking their prescribing behaviour with ABR may facilitate a change in practice, particularly among the GPs who routinely prescribe for UTI. Similar to other research, GPs would favour an intervention that would support their skills. ${ }^{25}$ Questioning prescribers also need scientific evidence to support their decisions, but in this instance, to reassure them that the actions are correct, and that the patient will be satisfied with the outcome. Change strategies should focus on the uncertainty of diagnosing UTIs within this grey area.
Patients need to be satisfied with the treatment, and GPs reassured that they were making adequate treatment decisions. The results revealed at least three profiles of patients, the young professional ('quick fixers'), the young mothers ('advice seekers'), and the mature patient ('experienced consulters'). Each type of patient can be satisfied differently from a 'simple' UTI consultation. For instance, the quick fixers, prioritise their personal health, adopt a low involvement approach and are satisfied to receive their antibiotic prescription. The advice seekers adopt a higher involvement perspective, discussing treatment options for their illness, an antibiotic is not a satisfactory outcome in all instances. Confirming the findings outlined in Leyton $e t a l,{ }^{26}$ patients with UTI do not always expect an antibiotic, instead they seek reassurance that their symptoms will improve. In this instance, time spent listening and interacting with patients may result in patient satisfaction with the consultations. ${ }^{27}$ Finally, the experienced consulters have experienced a UTI and antibiotic treatment in the past reinforcing the norm and expectations of treatment. The association between symptoms and treatment needs to be broken for change efforts to be successful in these cases. For all profiles of patients, the GP's decision-making power and influence hinges directly on the type of patient consulting for a UTI and vice versa. The findings indicated the interaction within the consultation and dialogue between the GP and the patient which activate the outcome.

\section{CONCLUSION}

This research provides insight into the decision-making processes contributing to the continued prescription and consumption of antibiotics for UTI. As a result of this qualitative research, behavioural interventions should focus on

1. Improving the quality of antibiotic prescribing for UTI by encouraging GPs to reflect on their current antibiotic-prescribing practices, including when they prescribe and what antibiotics they choose.

2. Supporting a dialogue between the GP and the patient within the consultation about the positive and negative aspects of antibiotic treatment for UTI particularly when symptoms are non-specific.

3. Integrating behavioural change messages into routine care without elongating the consultation.

Change will not emerge from a once-off intervention, however, steps can be taken to stimulate positive behaviour changes for both GPs and patients. Using these insights, Supporting the Improvement and Management of Prescribing for UTI (SIMPle), the complex intervention was designed using the principles of social marketing. SIMPle incorporated the following components: a professional development programme for the GP, which includes interactive workshops, audit and feedback reports on antibiotic prescribing and resistance, an electronic antibiotic prescribing prompt summarising guidelines, and a supportive framework educating patients on 
$\mathrm{ABR} .^{28}$ By integrating this intervention into routine care, the final intervention (SIMPle study) aimed to be sustainable and self-promoting. ${ }^{29}$

Author affiliations

${ }^{1}$ Discipline of General Practice, School of Medicine, National University of Ireland Galway, Galway, Ireland

${ }^{2}$ Discipline of Marketing, J.E. Cairnes School of Business and Economics, National University of Ireland Galway, Galway, Ireland

${ }^{3}$ Discipline of Economics, J.E. Cairnes School of Business and Economics, National University of Ireland Galway, Galway, Ireland

${ }^{4}$ Discipline of Bacteriology, School of Medicine, National University of Ireland Galway, Galway, Ireland

${ }^{5}$ Department of Medical Microbiology, University Hospital Galway, Galway, Ireland

${ }^{6}$ Department of Pharmacology \& Therapeutics, Trinity College Dublin, Ireland

Twitter Follow Sinead Duane at @smduane

Acknowledgements The authors would like to acknowledge the additional members of the SIMPle (Supporting the Improvement and Management of Prescribing for UTI) Study Team: Eamon O'Shea (Professor of Health Economics, Discipline of Economics, JE Cairnes School of Business and Economics and The Irish Centre for Social Gerontology, National University of Ireland, Galway, Ireland). They would like to thank the participants who helped with this research, and the gatekeepers who assisted with recruitment

Contributors SD, CD and AV conceived the study. SD and AC carried out the fieldwork. SD and $C D$ conducted the analysis. SD drafted the manuscript, and $\mathrm{CD}$ and $\mathrm{AV}$ revised the paper. All authors read and approved the final version of the manuscript. All members of the SIMPIe Study team contributed to the data collection and study design.

Funding This study was funded by the Health Research Board of Ireland under the Interdisciplinary Capacity Enhancement Award (ICE2011-10).

Competing interests None declared.

Ethics approval Ethical approval for the formative research data collection was obtained from the Irish College of General Practitioners.

Provenance and peer review Not commissioned; externally peer reviewed.

Data sharing statement No additional data are available.

Open Access This is an Open Access article distributed in accordance with the Creative Commons Attribution Non Commercial (CC BY-NC 4.0) license, which permits others to distribute, remix, adapt, build upon this work noncommercially, and license their derivative works on different terms, provided the original work is properly cited and the use is non-commercial. See: http:// creativecommons.org/licenses/by-nc/4.0/

\section{REFERENCES}

1. Vellinga A, Cormican M, Hanahoe B, et al. Antimicrobial management and appropriateness of treatment of urinary tract infection in general practice in Ireland. BMC Fam Prac 2011;12:108.

2. Wise R, Hart T, Cars O, et al. Antimicrobial resistance: is a major threat to public health. BMJ 1998;317:609-10.

3. Lipsitch M, Samore MH. Antimicrobial use and antimicrobial resistance: a population perspective. Emerg Infect Dis 2002;8:347-54.

4. Ashiru-Oredope D, Hopkins S, English Surveillance Programme for Antimicrobial Utilization and Resistance Oversight Group. Antimicrobial stewardship: English Surveillance Programme for Antimicrobial Utilization and Resistance (ESPAUR). J Antimicrob Chemother 2013;68:2421-3.

5. Arnold SR, Straus SE. Interventions to improve antibiotic prescribing practices in ambulatory care. Cochrane Database Syst Rev 2005;(4) CD003539.
6. Anomaly J. Collective action and individual choice: rethinking how we regulate narcotics and antibiotics. J Med Ethics 2013;39:752-6.

7. Chinburapa V, Larson LN, Brucks M, et al. Physician prescribing decisions: the effects of situational involvement and task complexity on information acquisition and decision making. Soc Sci Med 1993;36:1473-82.

8. McGowan B, Bennett $\mathrm{K}$, Casey MC, et al. Comparison of prescribing and adherence patterns of anti-osteoporotic medications post-admission for fragility type fracture in an urban teaching hospita and a rural teaching hospital in Ireland between 2005 and 2008. Ir J Med Sci 2013182:601-8.

9. Butler CC, Rollnick S, Pill R, et al. Understanding the culture of prescribing: qualitative study of general practitioners' and patients' perceptions of antibiotics for sore throats. BMJ 1998;317:637-42.

10. Rodrigues AT, Roque F, Falcão A, et al. Understanding physician antibiotic prescribing behaviour: a systematic review of qualitative studies. Int J Antimicrob Agents 2013;41:203-12.

11. Tonkin-Crine S, Yardley L, Little P. Antibiotic prescribing for acute respiratory tract infections in primary care: a systematic review and meta-ethnography. J Antimicrob Chemother 201166:2215-23.

12. Broom A, Broom J, Kirby E. Cultures of resistance? A Bourdieusian analysis of doctors' antibiotic prescribing. Soc Sci Med

2014;110:81-8.

13. Edgar T, Boyd SD, Palamé MJ. Sustainability for behaviour change in the fight against antibiotic resistance: a social marketing framework. J Antimicrob Chemother 2009;63:230-7.

14. Gupta K, Hooton TM, Stamm WE. Increasing antimicrobial resistance and the management of uncomplicated community-acquired urinary tract infections. Ann Intern Med 2001;135:41-50.

15. Vellinga A, Tansey S, Hanahoe B, et al. Trimethoprim and ciprofloxacin resistance and prescribing in urinary tract infection associated with Escherichia coli: a multilevel model. J Antimicrob Chemother 2012;67:2523-30.

16. Bishop MC. Uncomplicated Urinary Tract Infection. EAU Update Ser 2004:2:143-50.

17. Mazulli T. Diagnosis and management of simple and complicated urinary tract infections (UTIs). Can J Urol 2012;19 (Suppl 1):42-8.

18. Vellinga A, Murphy AW, Hanahoe B, et al. A multilevel analysis of trimethoprim and ciprofloxacin prescribing and resistance of uropathogenic Escherichia coli in general practice. J Antimicrob Chemother 2010;65:1514-20.

19. Prochaska JO, Velicer WF. The transtheoretical model of health behavior change. Am J Health Promot 1997;12:38-48.

20. Howard JA, Sheth JN. The theory of buyer behavior. New York: Wiley, 1969.

21. Truong VD, Garry T, Hall CM. Social marketing as the subject of doctoral dissertations. SMQ 2014;20:199-218.

22. Sobh R, Perry C. Research design and data analysis in realism research. E J Mark 2006;40:1194-209.

23. Braun V, Clarke V. Using thematic analysis in psychology. Qual Res Psychol 2006;3:77-101

24. Strandberg EL, Brorsson A, Hagstam C, et al. "I'm Dr Jekyll and Mr Hyde": are GPs' antibiotic prescribing patterns contextually dependent? A qualitative focus group study. Scand J Prim Health Care 2013;31:158-65.

25. Velasco E, Ziegelmann A, Eckmanns T, et al. Eliciting views on antibiotic prescribing and resistance among hospital and outpatient care physicians in Berlin, Germany: results of a qualitative study. BMJ Open 2012;2:e000398.

26. Leydon GM, Turner S, Smith $\mathrm{H}$, et al. Women's views about management and cause of urinary tract infection: qualitative interview study. BMJ 2010;340.

27. Lundkvist J, Åkerlind I, Borgquist L, et al. The more time spent on listening, the less time spent on prescribing antibiotics in general practice. Fam Pract 2002;19:638-40.

28. Duane $S$, Callan A, Galvin $S$, et al. Supporting the improvement and management of prescribing for urinary tract infections (SIMPle): protocol for a cluster randomized trial. Trials 2013;14:441.

29. Vellinga A, Galvin S, Duane S, et al. Intervention to improve the quality of antimicrobial prescribing for urinary tract infection: a cluster randomized trial. CMAJ 2015;187(17):150601. 